\title{
Short-term in situ warming influences early development of sessile assemblages
}

\author{
Dan A. Smale ${ }^{1, *}$, Thomas Wernberg ${ }^{1,2}$ \\ ${ }^{1}$ UWA Oceans Institute and School of Plant Biology, ${ }^{2}$ Australian Institute of Marine Science, University of Western Australia, \\ Crawley 60/09, Western Australia, Australia
}

\begin{abstract}
Increased temperature is arguably the most important facet of global climate change, as temperature influences processes across all biological scales. In terrestrial systems, the influence of warming on community dynamics has been investigated through field manipulations of temperature but, in contrast, there have been very few warming experiments conducted in the sea. Here, we used heated settlement panels to manipulate microhabitat temperature in situ for $>3 \mathrm{wk}$, to examine how short-term warming affects community development. We conducted 2 independent experiments in contrasting subtidal habitats in the Perth (Australia) metropolitan area, to determine the usefulness of the field-based approach and to examine consistencies in community-level response to warming. In the first experiment (Swan River estuary), a $\sim 2{ }^{\circ} \mathrm{C}$ warming treatment resulted in a lower space coverage of a tube-building amphipod and higher coverage of a solitary ascidian, Ciona intestinalis, which contributed to significant differences in community structure. In a second experiment (Hillarys Harbour), the bryozoan Watersipora subtorquata, spirorbid polychaete worms, and C. intestinalis covered less space on warmed surfaces than controls. This was associated with greater biomass of a colonial ascidian and widespread marine pest, Didemnum perlucidum, under warmer conditions, which overgrew and probably outcompeted other taxa. Our results show that community responses to short-term warming are variable and are influenced by individual responses of assemblage dominants. We discuss limitations of the study and highlight the importance of community-level, field-based manipulations of environmental change factors which examine interactions between all available members of the local species pool.
\end{abstract}

KEY WORDS: Hot plates - Temperature manipulations - Fouling assemblages · Climate change · Benthic community structure $\cdot$ Invasive species

Resale or republication not permitted without written consent of the publisher

\section{INTRODUCTION}

The surface waters of most oceans are warming and the frequency of extreme short-term thermal events (i.e. 'marine heat waves') is increasing (Solomon et al. 2007, Gille 2008). This has, and will continue to, impact upon human activities in the sea by influencing, amongst other things the distribution and quality of living resources (Perry et al. 2005, Cheung et al. 2010), ecosystem structure, resilience and services (Harley et al. 2006, Wernberg et al. 2011), and coastal industries, such as aquaculture, biofouling and tourism (Brander 2007, Poloczanska \& Butler 2010). Predicting the effects of sustained warming and other environmental change factors on marine biodiversity is a major undertaking for today's ecologists.

A key knowledge gap in marine ecology is that direction and magnitude of species' interactions varies under different environmental conditions (Callaway et al. 2002), yet our understanding of the effects of warming on processes such as predation, competition and facilitation remains poor (Harley et al. 2006). As such, attempts to predict changes in the distribu- 
tions of key species are currently hindered by limited knowledge of how warming will affect species interactions and other ecological processes in real habitats (Hawkins et al. 2008, Kordas et al. 2011). These uncertainties are best addressed by examining responses to warming at the community level under field conditions (Kordas et al. 2011).

Current knowledge of how seawater warming will affect marine organisms is largely based on laboratory and modeling approaches; there is a pressing need for relevant field manipulations that involve multiple, interacting species and environmental drivers (Harley et al. 2006, Wernberg et al. 2012). While laboratory experiments on the response of marine organisms to seawater warming yield useful information, they suffer from artificiality (Skelly 2002) and are not always of relevance to the 'real world' because: (1) they generally do not simulate natural variability in the (many) other environmental forces that shape ecological communities in nature; (2) they generally involve very few species, so that important interactions and processes may be missed; and (3) they often apply unrealistic treatments in terms of the rate or magnitude of warming.

In terrestrial ecosystems, ecologists have used polytunnels ('cloches') and fiberglass chambers to manipulate temperature in the field to study the effects of warming on community structure and ecological processes (e.g. Convey et al. 2002). In contrast, there have been very few warming experiments conducted in marine habitats (but see Morelissen \& Harley 2007). Most experimental work has utilized natural or manmade thermal gradients to correlate increased temperature with changes in species distributions or community structure. For example, Schiel et al. (2004) analysed a long-term dataset on the structure of benthic communities adjacent to thermal outfall from a power station and found significant, and unexpected, ecological effects of a $3.5^{\circ} \mathrm{C}$ temperature rise. Similar opportunistic studies have examined communitylevel responses to temporal thermal gradients driven by, for example, short-term extreme warming events (Dayton \& Tegner 1984, Garrabou et al. 2009). New techniques, however, can facilitate pre-planned, fully replicated, controlled manipulations of temperature in the field. Smale et al. (2011) found that a moderate temperature rise $\left(\sim 1^{\circ} \mathrm{C}\right)$ over warmed settlement panels led to significant increases in the space occupancy of an assemblage dominant. Here, we use the 'hot plate' methodology described by Smale et al. (2011) to experimentally examine, for the first time, community-level responses to in situ warming. We tested the hypothesis that a simulated short-term warming event would influence the early stages of sessile community development. We present data from 2 independent experiments that were conducted at different places and seasons. Without adequate replication at the experiment level it was not possible to make formal assessments of spatio-temporal variability in community responses to warming. Rather, it was our intention to examine the usefulness of the approach in 2 contrasting habitats and to make preliminary observations on the influence of warming on establishing sessile assemblages.

\section{MATERIALS AND METHODS}

\section{Study sites}

Experiments were conducted at 2 coastal study sites in Perth, Western Australia: the Swan River estuary and Hillarys Harbour (Fig. 1). The lower reaches of the Swan River are relatively wide and deep, and impacted by extensive development and industrialisation along the shoreline. The study site, towards the mouth of the estuary, is generally saline (mean salinity at $5 \mathrm{~m}$ depth in 2010 was 34 psu,

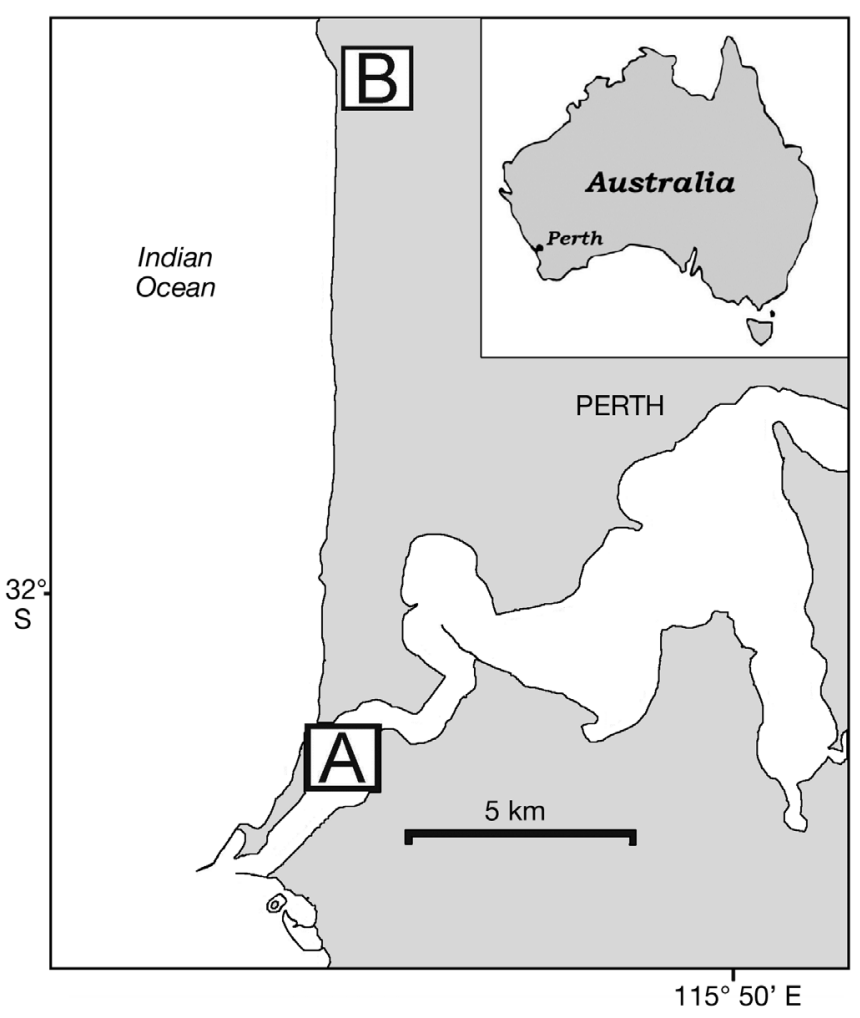

Fig. 1. Locations of the Swan River (A) and Hillarys Harbour (B) study sites in the Perth metropolitan area. Inset shows location of Perth on the Australian continent 
Swan River Trust, www.swanrivertrust.wa.gov.au) although freshwater input from persistent winter rains may reduce salinity on occasion, minimum weekly salinity in 2010 was 30 psu, Swan River Trust). The study site is characterised by semidiurnal tides that induce current speeds of up to $0.2 \mathrm{~m} \mathrm{~s}^{-1}$. The lower Swan River represents a dynamic and fluctuating thermal environment, as temperature may vary by up to $3.5^{\circ} \mathrm{C}$ within a single day (Smale et al. 2011). In general, the marine environment within Hillarys Harbour experiences less short-term variability in temperature, salinity, or current speed compared with the Swan River. For example, continuous hourly temperature measurements taken in 2010 showed that seawater temperature does not vary by more than $1.0^{\circ} \mathrm{C}$ in any one day, while salinity at $5 \mathrm{~m}$ depth does not fall below 35 psu (Australian Bureau of Meteorology, www.bom.gov.au). At both sites, monthly mean seawater temperature at $5 \mathrm{~m}$ depth typically varies from 15 to $24^{\circ} \mathrm{C}$ through the year.

\section{Temperature manipulations}

A heated-settlement panel array, comprising of 8 'hot plates' and 8 controls, was deployed at 3 to $5 \mathrm{~m}$ depth at both study sites to manipulate temperature in situ. Hot plates were similar in design to traditional settlement panels $(20 \times 20 \mathrm{~cm})$ except that the surface of the panel and the surrounding boundary layer of water were electrically heated to levels above ambient temperature. Warming was achieved by mounting an electrical heat trace, sealed in silicon, beneath an anodised aluminium plate. Power was supplied to the heat trace via a $50 \mathrm{~m}$ cable that connected to a shorebased control unit and mains power supply. The magnitude of heat transfer through the aluminium plate was a simple function of the applied voltage, which was calibrated prior to deployment. In this way, the substratum available for colonisation and growth, and a boundary layer of water (typically 4 to $12 \mathrm{~mm}$ in thickness at water flow speeds encountered at the study sites, see Smale et al. 2011), were warmed in situ for $>3$ wk at both study sites. We aimed to sustain a mean warming treatment of $\sim 2^{\circ} \mathrm{C}$, as hard-bottom coastal habitats in the region experience this magnitude of warming during short-term thermal events (Smale \& Wernberg 2009, Smale \& Wernberg 2012).

During the deployments, an experimental settling surface (woven shade cloth, $2 \mathrm{~mm}$ thick) was fixed to the aluminium plate to serve as substratum for colonisation by marine organisms. Pilot studies showed that the shade cloth facilitated uniform, con- stant diffusion of warm water from the heated metal plate to the surrounding water layer, while providing a suitable surface for rapid colonisation by marine organisms. Control plates were identical in design to hot plates, but remained at ambient temperature. Stainless steel T-type needle probes were mounted $2 \mathrm{~mm}$ above the centre of 2 control and 2 hot plates, to record temperature every $6 \mathrm{~s}$ for the duration of the experiments. Controls and hot plates were mounted alternately across the frame, $20 \mathrm{~cm}$ apart. At both sites the array was deployed with plates facing downwards (to select for fauna rather than flora) and suspended 1.5 to $2.0 \mathrm{~m}$ above the seabed. The Swan River and Hillarys Harbour experiments were conducted in September 2010 and March 2011, respectively. Following each deployment, the shade cloth surfaces were removed from the array and returned to the laboratory. All sessile fauna and flora $>5 \mathrm{~mm}$ was identified to the lowest taxonomic level possible (usually species but occasionally distinct morphotypes), and the percent cover of each taxon was recorded for each plate by overlaying a transparent grid and quantifying spatial coverage.

\section{Analysis}

Daily mean temperatures were calculated for both hot plates and controls for each deployment (means of 2 loggers per treatment and 240 observations per day). The effect of warming on assemblage structure was analysed with multivariate statistics, using PRIMER 6 software (Clarke \& Warwick 2001, Anderson et al. 2007). The percent cover of all taxa were $\log (x+1)$ transformed to down-weight the relative influence of dominant space occupiers before constructing Bray-Curtis similarity matrices. MDS ordinations for each experiment were used to visualise partitioning in assemblage structure between the hot plates and controls. PERMANOVA tests indicated the significance of any dissimilarity between treatments (with 'treatment' as a fixed factor, and 999 unrestricted permutations of raw data). Where significant differences were detected ( $p<0.05)$, a SIMPER analysis was conducted to determine which taxa were the principal contributors to the observed dissimilarity in assemblage structure. PERMDISP, which examines differences in multivariate dispersion within groups, was used to assess differences in variability within hot plate and control treatments. Furthermore, the effect of warming on the 4 dominant space occupiers in each experiment was examined in detail. Plots of mean percent cover $(n=8)$ on hot plates 
and controls were constructed for each experiment. Significance tests were conducted with univariate PERMANOVA, by constructing similarity matrices based on Euclidian distances of untransformed percent cover data (again, with 'treatment' as a fixed factor, and 999 unrestricted permutations of raw data). Finally, univariate PERMANOVA (using the same approach as described above for individual taxa) was used to test for differences in assemblage level metrics (i.e. total biomass and taxon richness) between hot plates and controls.

\section{RESULTS}

Warming treatments of $\sim 2^{\circ} \mathrm{C}$ were maintained for $32 \mathrm{~d}$ in the Swan River and $22 \mathrm{~d}$ in Hillarys Harbour (Fig. 2). The Swan River deployment was conducted in late winter, so that ambient seawater temperatures were low $\left(\sim 16\right.$ to $\left.18^{\circ} \mathrm{C}\right)$ in comparison to the summer deployments at Hillarys Harbour ( 24 to $\left.27^{\circ} \mathrm{C}\right)$. Overall, warming treatments reflected natural variability in seawater temperature adequately, although the magnitude of warming increased slightly during the Swan River experiment but decreased throughout the deployment in Hillarys Harbour (Fig. 2). Variability in the daily mean warming treatment between replicate hot plates was generally low (average difference in daily means between replicate hot plates at Swan River: $0.44 \pm 0.06$ and at Hillarys Harbour: $0.33 \pm 0.05$ ), although a maximum disparity of $\sim 1^{\circ} \mathrm{C}$ was recorded during the Swan River experiment (Fig. 2a,b).

Sessile assemblages comprised relatively few taxa (8 at the Swan River and 10 at Hillarys Harbour) but were strikingly different in structure between study

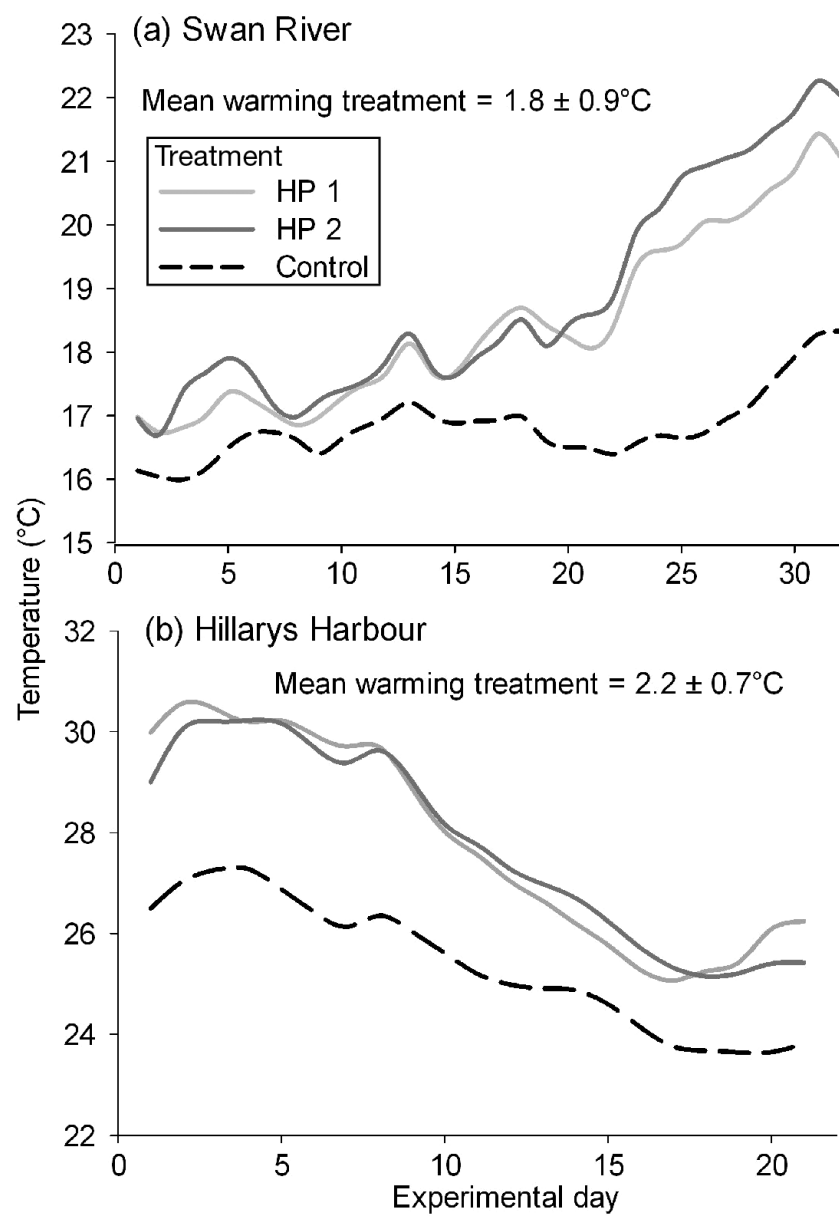

(c) Swan River: $F_{1,14}=5.83, p=0.003$

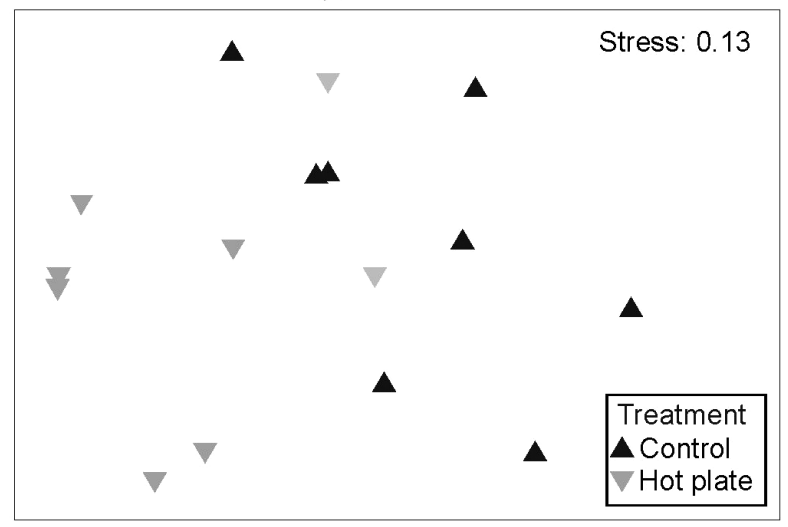

(d) Hillarys Harbour: $F_{1,14}=9.48, p=0.002$

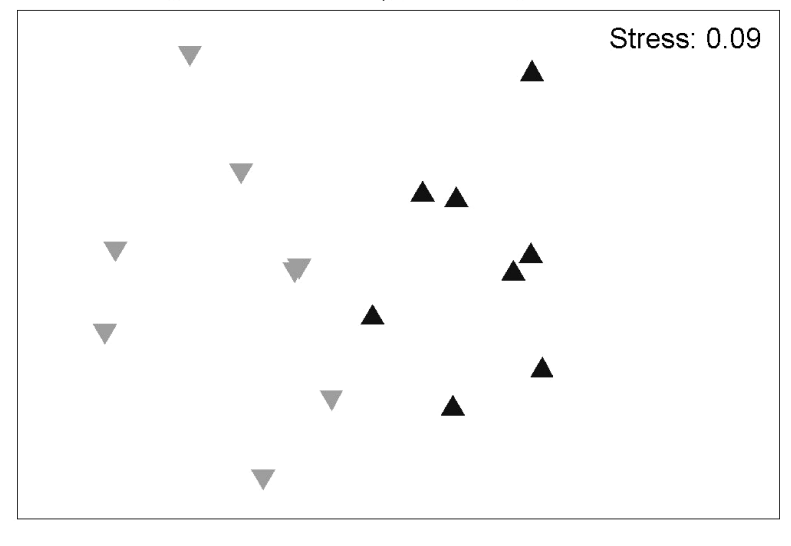

Fig. 2. Daily mean temperature over 2 independent hot plates (HP1 and HP2) and over a control plate during the (a) Swan River and (b) Hillarys Harbour experiments. The mean warming treatment $( \pm$ SD) applied throughout each experiment is also given. MDS ordinations show sessile assemblage structure on hot plates and controls following the (c) Swan River and (d) Hillarys Harbour deployments, which include results of PERMANOVAs to test for differences in multivariate assemblage structure between treatments 
sites. Swan River assemblages were dominated by a tube-building crustacean and hydroids, whereas assemblages at Hillarys Harbour were characterised by very high space coverage of the colonial ascidian Didemnum perlucidum, which occupied up to $95 \%$ of the plates' surface. Didemnum perlucidum colonies often overgrew other taxa, such as the cheilostome bryozoan Watersipora subtorquata and spirorbid polychaetes. The identity of $D$. perlucidum was confirmed through both expert taxonomic advice and DNA barcoding techniques (Smale \& Childs 2012). In general, taxa were encrusting or prostrate and remained within the warmed boundary layer for the duration of the experiments (with the exception of some of the larger individuals of the solitary ascidian Ciona intestinalis and hydroids).

For each experiment, MDS ordinations indicated partitioning in the structure of sessile assemblages on hot plates and controls, although this was more pronounced at Hillarys Harbour (Fig. 2c,d). We used PERMANOVA to detect highly significant differences in multivariate assemblage structure between hot plates and controls in both experiments (Swan River: $F_{1,4}=5.83$, $\mathrm{p}=0.003$, Hillarys Harbour: $F_{1,4}=9.48$, $\mathrm{p}=0.002$, Fig. 2). PERMDISP detected no significant differences in withingroup variability (Swan River: $F_{1,4}=$ 0.05, $\mathrm{p}=0.80$, Hillarys Harbour: $F_{1,4}=$ $0.47, \mathrm{p}=0.58$ ). The difference in multivariate structure between hot plates and controls in the Swan River was principally driven by a tube-building amphipod (a member of the Ischyroceridae family), which occupied more space on controls than hot plates, and also by Ciona intestinalis and hydroids, both of which covered more space on hot plates than controls (Table 1). In the Hillarys Harbour experiment, differences were driven by the cheilostome bryozoan Watersipora subtorquata, spirorbid worms and $C$. intestinalis, which all covered more space on controls than hot plates, and by hydroids, which occupied more space on hot plates than controls (Table 1).
Mean percent cover of individual taxa on hot plates and controls showed inconsistencies in responses to the warming treatment, both between taxa and between deployments (Fig. 3). For example, in the Swan River warming caused a significant increase in the percent cover of Ciona intestinalis (PERMANOVA: $\left.F_{1,14}=6.43, \mathrm{p}=0.025\right)$, while the other dominant taxa, the Ischyrocerid amphipod, hydroids and Watersipora subtorquata, exhibited no response to warming

Table 1. Percentage contributions of individual species to observed differences between control and hot plate assemblages, for both the Swan River and Hillarys Harbour, as determined by SIMPER analysis. Percent cover data were $\log (x+1)$ transformed prior to analysis. Only the top 4 contributors to dissimilarities are shown. Average dissimilarities between treatments are given in parentheses. Control: mean cover on control panels $(n=8)$; Hot plate: mean cover on hot plates $(n=8)$; Diss/SD: average dissimilarity divided by standard deviation; Contri.: contribution of each species to differences between treatments; Cum.: running total of the contribution to the observed dissimilarity

\begin{tabular}{|lccccc|}
\hline \multicolumn{7}{|c}{$\begin{array}{c}\text { Control } \\
(\%)\end{array}$} & $\begin{array}{c}\text { Hot plate } \\
(\%)\end{array}$ & $\begin{array}{c}\text { Diss/SD } \\
\text { Contri. } \\
(\%)\end{array}$ & $\begin{array}{c}\text { Cum. } \\
(\%)\end{array}$ \\
\hline \multicolumn{2}{l}{ Hillarys Harbour (control vs. hot plate $\mathbf{= 1 8 \% )}$} & & & \\
Ischyrocerid amphipod & 3.12 & 2.49 & 1.41 & 27.94 & 27.94 \\
Ciona intestinalis & 0.67 & 1.32 & 1.37 & 25.43 & 53.38 \\
Hydroids & 2.67 & 2.86 & 1.21 & 21.38 & 74.76 \\
Botrylloides cf. perspicuus & 0.00 & 0.43 & 1.27 & 15.09 & 89.85 \\
Hillarys Harbour (control vs. hot plate $\mathbf{1 8} \%)$ & & & \\
Watersipora subtorquata & 1.75 & 1.00 & 1.40 & 18.72 & 18.72 \\
Spirorbids & 1.03 & 0.35 & 2.86 & 16.48 & 35.20 \\
Hydroids & 2.09 & 2.58 & 1.53 & 15.46 & 50.66 \\
Ciona intestinalis & 0.88 & 0.00 & 1.55 & 11.76 & 62.42 \\
\hline
\end{tabular}

(a) Swan River

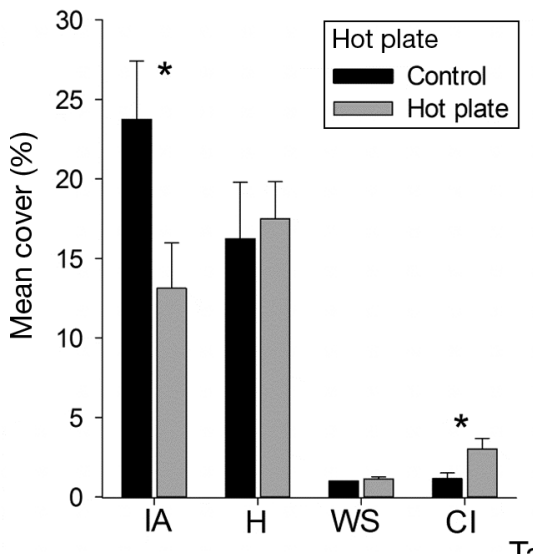

Taxa (b) Hillarys Harbour

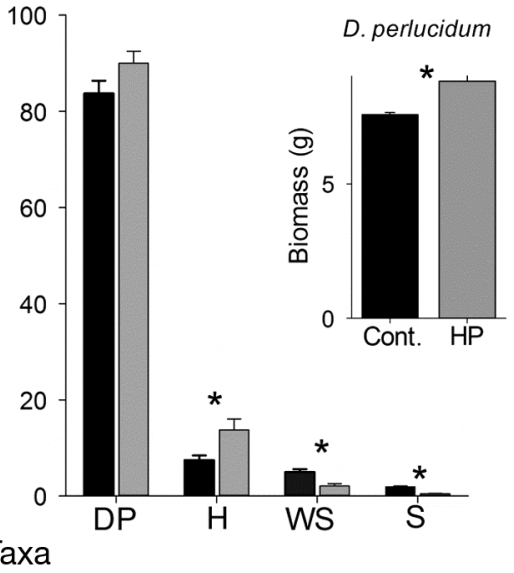

Fig. 3. Mean $( \pm \mathrm{SE})$ percent cover $(\mathrm{n}=8)$ of the dominant space occupiers on both hot plates (HP) and controls (cont.), following deployments in (a) the Swan River and (b) Hillarys Harbour. IA = Ischyrocerid amphipod, H = Hydroids, WS $=$ Watersipora subtorquata, $\mathrm{CI}=$ Ciona intestinalis, DP $=$ Didemnum perlucidum, $\mathrm{S}=$ Spirorbids. A significant difference at $\mathrm{p}<0.05$ is denoted by *. Mean total dry biomass of Didemnum perlucidum on hot plates and controls is also shown for Hillarys Harbour 
(Fig. 3). Conversely, at Hillarys Harbour, the space coverage of these taxa did vary between treatments, with hydroids increasing (PERMANOVA: $F_{1,14}=6.51$, $\mathrm{p}=0.048$ ) and $W$. subtorquata decreasing (PERMANOVA: $F_{1,14}=14.29, \mathrm{p}=0.003$ ) in cover on hot plates compared with controls. Spirorbid worms were also significantly more abundant on control plates (PERMANOVA: $F_{1,14}=47.77, \mathrm{p}=0.001$ ). At Hillarys Harbour, all plates were dominated by Didemnum perlucidum, which covered 70 to $95 \%$ of every plate. Mean percent cover of $D$. perlucidum was slightly greater on hot plates but this difference was not statistically significant. However, D. perlucidum colonies on hot plates were observed to be generally more developed and 'thicker', in terms of densities of zooids, than on controls. Biomass (dry weight) of $D$. perlucidum was analysed to examine this further; biomass on hot plates was significantly greater than on controls (Fig. 3, PERMANOVA: $\left.F_{1,14}=16.28, \mathrm{p}=0.001\right)$. Finally, total species richness varied with treatment, but the direction of change differed between experiments (Fig. 4). In the Swan River experiment, mean richness was greater on hot plates compared with controls (PERMANOVA: $\left.F_{1,14}=14.01, \mathrm{p}=0.002\right)$, whereas at Hillarys Harbour mean richness was lower on hot plates compared with controls (PERMANOVA: $F_{1,14}=$ 19.70, $\mathrm{p}=0.001$ ).

\section{DISCUSSION}

Microhabitat warming of $\sim 2^{\circ} \mathrm{C}$ caused significant shifts in the structure of sessile assemblages on hard

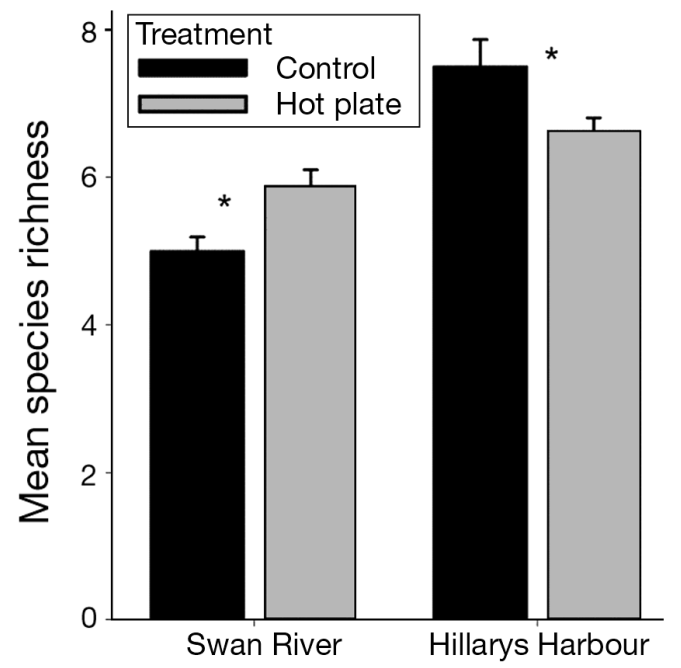

Fig. 4. Mean $( \pm \mathrm{SE})$ total species richness $(\mathrm{n}=8)$ on both hot plates and controls at the Swan River and Hillarys Harbour. A significant difference at $\mathrm{p}<0.05$ is denoted by * substrata in both experiments, which supported our hypothesis that increased temperature would alter early community development. We also observed pronounced between-taxa variability in the direction and magnitude of response to warming. To our knowledge, these were the first fully replicated, preplanned controlled manipulations of in situ seawater temperature, which experimentally demonstrated that short-term warming affects early community development.

In the Swan River, warming led to a significant decrease in the space coverage of a tube-building Ischyrocerid amphipod but an increase in space occupancy of the widespread solitary ascidian Ciona intestinalis, both of which were principal discriminators of overall assemblage structure between hot plates and controls. Ciona intestinalis is a globally distributed marine pest, which can suppress local species richness and cause major problems for aquaculture (Blum et al. 2007, Daigle \& Herbinger 2009). In this experiment, we suggest that the higher space coverage on hot plates was primarily a consequence of larger individuals, as the number of recruits did not appear to differ between treatments (D. A. Smale \& T. Wernberg pers. obs.). Previous work has shown that, within the 15 to $25^{\circ} \mathrm{C}$ temperature range, warmer waters induce faster growth rates in C. intestinalis (Yamaguchi 1975), which could explain the greater space occupancy on the warmed surfaces. Interestingly, mean species richness on hot plates was greater than on controls in the Swan River, principally because of more frequent occurrences of the ascidians C. intestinalis and Botrylloides cf. perspicuus, which is probably an indigenous colonial ascidian with tropical affinity (Kott 1985). Further work is needed to determine whether these ascidians will thrive under warmer conditions, but research conducted in North America has suggested that increased water temperature, in combination with coastal eutrophication, is leading to greater local abundances, higher invasion success and facilitating range expansions of members of these genera (Stachowicz et al. 2002, Lambert \& Lambert 2003, Sorte et al. 2010, Sorte \& Stachowicz 2011).

At Hillarys Harbour, we recorded greater space coverage of hydroids and lower space coverage of Watersipora subtorquata and spirorbid worms on hot plates compared with controls. Furthermore, the ascidian Ciona intestinalis, which covered less space on hot plates than controls (in contrast to the Swan River experiment), was a principal contributor to the observed difference in multivariate assemblage composition between treatments. While further manipulation is required, we suggest that the response of the 
assemblage dominant Didemnum perlucidum to warming could (at least partly) explain the reduced species richness and lower space occupancy of Watersipora subtorquata, Ciona intestinalis and spirorbid worms on the heated panels.

The colonial ascidian Didemnum perlucidum occupied $>70 \%$ of free space on all panel surfaces at Hillarys Harbour. Space coverage was slightly (but not significantly) greater on hot plates than controls, while biomass of $D$. perlucidum was significantly greater on warmed surfaces, seemingly because of thicker, more developed colonies. Didemnum perlucidum is widespread in tropical waters and is currently thought to be increasing in abundance and distribution in South America and throughout the Indo-West Pacific (Lambert 2002, Kremer et al. 2010). The occurrence of $D$. perlucidum in Australia has only recently been confirmed (Smale \& Childs 2012), but it is widespread through the Indo-Pacific and could be undergoing a range expansion in response to increased shipping, temperature, and eutrophication (Lambert 2002, 2007). Crucially, we observed $D$. perlucidum overgrowing other taxa, as has been recorded elsewhere (Lambert 2002, but see Kremer \& Rocha 2011), particularly on the heated panels where colonies were notably more developed. In general, at Hillarys Harbour fewer taxa were inter-

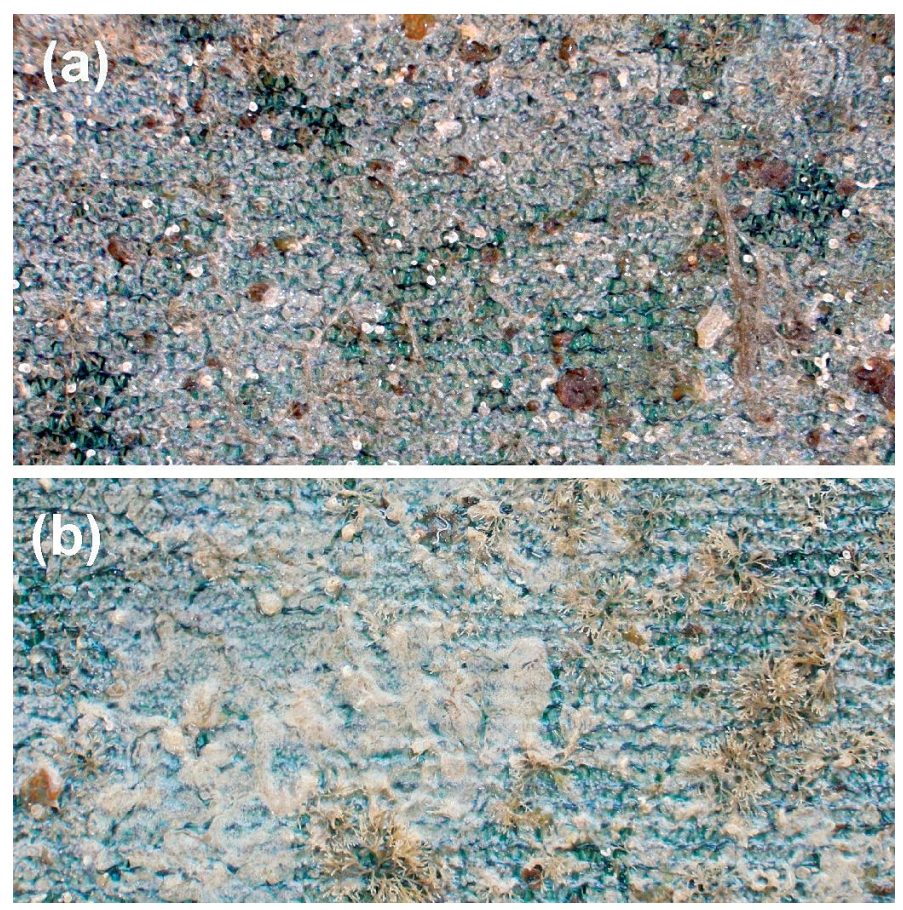

Fig. 5. A typical sessile assemblage on (a) a control and (b) a hot plate following the $22 \mathrm{~d}$ deployment in Hillarys Harbour spersed with $D$. perlucidum colonies on hot plates compared with controls (Fig. 5), resulting in lower overall species richness. Furthermore, in the previous hot plate experiment, which was conducted in the Swan River during a period of $D$. perlucidum recruitment, Smale et al. (2011) recorded higher space occupancy of this species on hot plates compare with controls. It is therefore plausible that our warming treatment induced either earlier recruitment or faster development/growth of $D$. perlucidum, which in turn influenced community development and species richness by monopolising space and overgrowing other organisms. However, controlled manipulations of this species (in conjunction with warming treatments) are needed to explicitly examine its structuring role in sessile assemblages. Moreover, exactly how temperature affected the development of $D$. perlucidum colonies - through increased metabolism, larval settlement behavior, or timing of recruitment - requires clarification.

The hot plate system represents a useful tool for conducting short-term warming experiments in the marine environment. Clearly, warming only the substratum and surrounding boundary layer of seawater does not fully simulate climate change scenarios or 'real' heat waves, which occur at much greater spatial scales (see Smale et al. 2011 for further discussion). This field approach, however, does remove much of the artificiality associated with laboratory experiments (which generally involve few species and do not mimic natural environmental variability) to facilitate experiments on the effects of warming on processes such as recruitment, growth, and species interactions. Here, we maintained a warming treatment of $\sim 2^{\circ} \mathrm{C}$, which was ecologically meaningful in both magnitude and capacity to track natural variability for over 3 weeks in the field. By examining the impacts of warming on whole communities in situ, we were able to record (unexpected) interactions between a range of species from the local pool, which can be used to direct further research, including focused laboratory experiments.

Acknowledgements. We thank R. Scott for technical assistance with the hot plate system and S. Childs, K. Waddington and T. Langlois for assistance in the field. We sincerely thank staff at the North Fremantle Water Police Facility and Hillarys Harbour for supporting and hosting the research. The Swan River Trust (Government of Western Australia) provided monitoring data from the Swan River estuary. This work was funded by a University of Western Australia Research Development Award to D.A.S. 


\section{LITERATURE CITED}

Anderson MJ, Gorley RN, Clarke KR (2007) Permanova+ for primer: guide to software and statistical methods. PRIMER-E, Plymouth

Blum JC, Chang AL, Liljesthröm M, Schenk ME, Steinberg MK, Ruiz GM (2007) The non-native solitary ascidian Ciona intestinalis (L.) depresses species richness. J Exp Mar Biol Ecol 342:5-14

Brander KM (2007) Global fish production and climate change. Proc Natl Acad Sci USA 104:19709-19714

Callaway RM, Brooker RW, Choler P, Kikvidze Z and others (2002) Positive interactions among alpine plants increase with stress. Nature 417:844-848

> Cheung WWL, Lam VWY, Sarmiento JL, Kearney K, Watson REG, Zeller D, Pauly D (2010) Large-scale redistribution of maximum fisheries catch potential in the global ocean under climate change. Glob Change Biol 16:24-35

Clarke KR, Warwick RM (2001) Change in marine communities: an approach to statistical analysis and interpretation. PRIMER-E, Plymouth

> Convey P, Pugh PJA, Jackson C, Murray AW, Ruhland CT, Xiong FS, Day TA (2002) Response of Antarctic terrestrial microarthropods to long-term climate manipulations. Ecology 83:3130-3140

- Daigle RM, Herbinger CM (2009) Ecological interactions between the vase tunicate (Ciona intestinalis) and the farmed blue mussel (Mytilus edulis) in Nova Scotia, Canada. Aquat Invasions 4:177-187

> Dayton PK, Tegner MJ (1984) Catastrophic storms, El Niño, and patch stability in a southern California kelp community. Science 224:283-285

> Garrabou J, Coma R, Bensoussan N, Bally M and others (2009) Mass mortality in Northwestern Mediterranean rocky benthic communities: effects of the 2003 heat wave. Glob Change Biol 15:1090-1103

Gille ST (2008) Decadal-scale temperature trends in the Southern Hemisphere ocean. J Clim 21:4749-4765

Harley CDG, Randall Hughes A, Hultgren KM, Miner BG and others (2006) The impacts of climate change in coastal marine systems. Ecol Lett 9:228-241

Hawkins SJ, Moore PJ, Burrows MT, Poloczanska E and others (2008) Complex interactions in a rapidly changing world: responses of rocky shore communities to recent climate change. Clim Res 37:123-133

> Kordas RL, Harley CDG, O'Connor MI (2011) Community ecology in a warming world: the influence of temperature on interspecific interactions in marine systems. J Exp Mar Biol Ecol 400:218-226

Kott P (1985) The Australian Ascidiacea. Part 1. Phlebobranchia and Stolidobranchia. Mem Queensl Mus 23: $1-440$

Kremer LP, Rocha RM (2011) The role of Didemnum perlucidum F. Monniot, 1983 (Tunicata, Ascidiacea) in a marine fouling community Aquat Invas 6:441-449

> Kremer LP, Rocha RM, Roper JJ (2010) An experimental test of the colonization ability in the potentially invasive Didemnum perlucidum (Tunicata, Ascidiacea). Biol Invasions 12:1581-1590

> Lambert G (2002) Nonindigenous ascidians in tropical waters. Pac Sci 56:291-298

Editorial responsibility: Richard Osman,

Edgewater, Maryland, USA
Lambert G (2007) Invasive sea squirts: a growing global problem. J Exp Mar Biol Ecol 342:3-4

Lambert CC, Lambert G (2003) Persistence and differential distribution of nonindigenous ascidians in harbors of the Southern California Bight. Mar Ecol Prog Ser 259:145-161

Morelissen B, Harley CDG (2007) The effects of temperature on producers, consumers, and plant-herbivore interactions in an intertidal community. J Exp Mar Biol Ecol 348: 162-173

Perry AL, Low PJ, Ellis JR, Reynolds JD (2005) Climate change and distribution shifts in marine fishes. Science 308:1912-1915

Poloczanska ES, Butler AJ (2010) Biofouling and climate change. In: Dürr S, Thomason JC (eds) Biofouling. WileyBlackwell, Oxford, p 333-347

Schiel DR, Steinbeck JR, Foster MS (2004) Ten years of induced ocean warming causes comprehensive changes in marine benthic communities. Ecology 85:1833-1839

Skelly DK (2002) Experimental venue and estimation of interaction strength. Ecology 83:2097-2101

Smale DA, Childs S (2012) The occurrence of a widespread marine invader, Didemnum perlucidum (Tunicata, Ascidiacea) in Western Australia. Biol Invasions (in press) doi: 10.1007/s10530-011-0167-8

Smale DA, Wernberg T (2009) Satellite-derived SST data as a proxy for water temperature in nearshore benthic ecology. Mar Ecol Prog Ser 387:27-37

Smale DA, Wernberg T (2012) Ecological observations associated with an anomalous warming event at the Houtman Abrolhos Islands, Western Australia. Coral Reefs (in press) doi: 10.1007/s00338-012-0873-4

> Smale DA, Wernberg T, Peck LS, Barnes DKA (2011) Turning on the heat: ecological response to simulated warming in the sea. PLoS ONE 6:e16050

Solomon S, Qin D, Manning M, Chen Z and others (2007) Contribution of working group 1 to the fourth assessment report of the IPCC. Cambridge University Press, Cambridge

Sorte CJB, Stachowicz JJ (2011) Patterns and process of compositional change in a California epibenthic community. Mar Ecol Prog Ser 435:63-74

Sorte CJ, Williams S, Zerebecki R (2010) Ocean warming increases threat of invasive species in a marine fouling community. Ecology 91:2198-2204

> Stachowicz JJ, Terwin JR, Whitlatch RB, Osman RW (2002) Linking climate change and biological invasions: ocean warming facilitates nonindigenous species invasions. Proc Natl Acad Sci USA 99:15497-15500

Wernberg T, Russell BD, Moore PJ, Ling SD and others (2011) Impacts of climate change in a global hotspot for temperate marine biodiversity and ocean warming. J Exp Mar Biol Ecol 400:7-16

Wernberg T, Smale DA, Thomsen MS (2012) A decade of climate change experiments on marine organisms: procedures, patterns and problems. Glob Change Biol 18: 1491-1498

Yamaguchi M (1975) Growth and reproductive cycles of the marine fouling ascidians Ciona intestinalis, Styela plicata, Botrylloides violaceus and Leptoclinum mitsukuriiat Aburatsubo-Moroiso Inlet (central Japan). Mar Biol 29:253-259

Submitted: December 9, 2012; Accepted: February 16, 2012 Proofs received from author(s): April 23, 2012 